\title{
EL EFECTO DE LA ATENCIÓN INFANTIL SUBSIDIADA SOBRE LA PARTICIPACIÓN DE LA MUJER EN EL MERCADO LABORAL DE COSTA RICA.
}

\section{THE EFFECT OF SUBSIDIZED CHILDCARE ON FEMALE LABOR MARKET PARTICIPATION IN COSTA RICA.}

\author{
Aníbal Jesús Quirós ${ }^{1}$ \\ Juan Robalino ${ }^{2}$
}

\begin{abstract}
En este estudio se estima el efecto de la atención infantil sobre la probabilidad de participación en el mercado laboral y sobre las horas trabajadas de las mujeres, utilizando las encuestas de hogares y una combinación de técnicas econométricas. Se encontró que las mujeres que pertenecen a hogares que se benefician de los servicios de cuido, en comparación con las que no, tienen entre 9 y 14 puntos porcentuales más de probabilidad de participar en la fuerza laboral. También, para el grupo de mujeres ocupadas, el número de horas trabajadas es entre un 8 y 19 por ciento más alto para las que se benefician del cuido. Los efectos sobre la participación son mayores para las mujeres que no han terminado la secundaria. La evidencia también sugiere que los efectos sobre las horas trabajadas son mayores para las mujeres del quintil más pobre. La expansión de centros de cuido podría contribuir a cerrar la brecha de género en la oferta laboral, en las mujeres de menores ingresos. Esto puede tener efectos importantes en la reducción de las tasas de pobreza.
\end{abstract}

PALABRAS CLAVE: CUIDO INFANTIL, RED DE CUIDO INFANTIL, PARTICIPACION LABORAL FEMENINA, HORAS DE TRABAJO FEMENINO

CLASIFICACIÓN JEL: J21, J22, J78

1 Universidad de Costa Rica, Escuela de Economía; Código Postal: 11501-2060; San José, Costa Rica; anibal.quiros@ucr.ac.cr 


\begin{abstract}
In this study, we estimate the effects of the use of subsidized childcare on the probability of female participation in the labor market and on the numbers of hours worked in Costa Rica. We use household surveys and a combination of econometric techniques in order to control for potential bias. We find that females that belong to beneficiary households have between 9 and 14 additional percentage points in the probability to participate in the labor force. They also worked between 8 and 19 percent more than females that are not in the program. The effects on labor participation are larger for women that have not finished high school. The effects on the numbers of hours worked are larger for those in the poorest quintile. These results suggest that the expansion of this program can contribute to reduce the labor participation gender gap, which is larger among the poor. This, in turn, could lead to important reductions in poverty levels.
\end{abstract}

KEYWORDS: CHILD CARE, LABOR MARKET PARTICIPATION, FEMALE LABOR FORCE PARTICIPATION

JEL CLASSIFICATION: J21, J22, J78

\title{
I. INTRODUCCIÓN
}

La tasa de inactividad de las mujeres en Costa Rica, a pesar de que se ha venido reduciendo en las últimas décadas (Jiménez-Fontana, 2016), se mantiene en niveles altos. Para aquellas en los hogares de ingresos más bajos, por ejemplo, la tasa de inactividad ha alcanzado el $70 \%$ (Robalino et al., 2019). Si las mujeres de los sectores más pobres se incorporan a la fuerza laboral, la tasa de pobreza se reduciría significativamente (Programa Estado de la Nación [PEN], 2018). Se ha argumentado que una de las barreras más grandes a las que se enfrentan las mujeres para poder insertarse en el mercado laboral es la falta de alternativas de cuido (Jiménez-Fontana, 2016). Políticas públicas para resolver este problema se han implementado en diferentes lugares del mundo y Costa Rica no es la excepción. En el año 2010, se implementó el programa Red de Cuido, que actualmente cubre cerca de 60 mil menores (UNICEF Costa Rica, 2020). Sin embargo, debido a los retos de focalización (PEN, 2019) y a la presencia de otras barreras a la inserción, no es claro que estas medidas tengan el efecto deseado.

En esta investigación se estudia si el programa subsidiado de cuido infantil de Costa Rica ha tenido efecto sobre la participación en el mercado laboral en las mujeres de los hogares beneficiados. Este tipo de programas sociales puede aliviar la presión generada por la duplicación de labores que se recargan en las mujeres ocupadas, quienes deben cumplir con las funciones propias de su puesto y "las obligaciones" de cuido en su hogar (Beneria, 2007). Estas presiones se acentúan con el incremento de hogares con jefatura femenina y los bajos niveles de logro educativo de los grupos vulnerables (Porras, 2012; Programa Estado de la Nación, 2010).

Existe evidencia previa sobre los efectos de políticas de cuido en otros países. Impactos positivos sobre la participación laboral se han encontrado en México (Ángeles et al., 2011), Ecuador (Rosero \& Oosterbeek, 2011), Colombia (Attanasio \& Vera-Hernández, 2004), Argentina (Berlinski \& Galiani, 2007), Alemania (Haan \& Wrohlich, 2009), Hungría (Lovász \& Szabó-Morvai, 2013) y Canadá (Baker et al., 2008). Sin embargo, en Chile (Encina Galaz \& Martínez Alvear, 2009) y en Uruguay (Nollenberger \& Perazzo, 2016) se encuentra que el impacto del cuido sobre la fuerza laboral es nulo.

Concretamente, en este estudio, se busca estimar los efectos de los servicios brindados por dos alternativas que atienden menores subsidiados por el IMAS (Instituto Mixto de Ayuda Social), los Hogares Comunitarios (HC) o los Centros de Cuido y de Atención Infantil (CECUDI), 
que pertenecen a la Red de Cuido (RECUDI), sobre la participación laboral y las horas trabajadas de las mujeres cuyos hogares recibieron el beneficio durante el período 2016-2019. Para ello, se utilizan la Encuesta Nacionales de Hogares (ENAHO) del Instituto Nacional de Estadística y Censos (INEC) que poseen datos de las características laborales de la mujer y del hogar y si utilizan o no los programas de cuido mencionados anteriormente.

Estimar los efectos del cuido sobre la participación en el mercado laboral presenta retos importantes. La presencia de variables que simultáneamente afectan las decisiones del mercado laboral y las decisiones de uso de cuido podrían sesgar las estimaciones. Estos sesgos se pueden ver acentuados por los procesos de autoselección en la decisión de participación en este tipo de programas (Encina Galaz \& Martínez Alvear, 2009).

Nuestra contribución a la literatura se da en dos aspectos. Primero, este es de los primeros estudios, sino el primero, que responde esta pregunta de investigación en Costa Rica con métodos de evaluación de impacto quasi-experimentales. Aunque existe evidencia para otros países de América Latina, no se puede asegurar que los mismos resultados se presenten en Costa Rica debido a las diferencias del contexto. Así, en este estudio se genera información clave para el mejoramiento de la política pública. Segundo, exploramos variaciones en el impacto del cuido utilizando características de las mujeres. Es de esperar que los impactos de una política no sean los mismos para toda la población, sin embargo, esto no se ha explorado en estudios previos. Esto es importante para diseñar un programa en forma más focalizada y generar una mejor relación costo-efectividad del programa.

Para resolver estos retos, se siguen tres pasos. Primero, a priori, se eliminan de la muestra mujeres que no pertenecen a localidades donde había mujeres utilizando los servicios de cuido. Esto nos permite comparar solamente mujeres que utilizan cuido con mujeres que no utilizan cuido de la misma comunidad. Es de esperar que aquellas mujeres que pertenecen a las mismas comunidades tengan características, inclusive no observables, similares. Segundo, se utiliza la técnica Propensity Score Matching (PSM) para mejorar el balance en las características observables entre las mujeres que utilizan servicios de cuido y aquellas que no. Tercero, con la muestra resultante, se implementa Mínimos Cuadrados Ordinarios (MCO) y los métodos logit binomial, logit multivariado y tobit para eliminar desbalances remanentes luego del PSM.

Los resultados de esta investigación demuestran que el cuido subsidiado favorece la participación de las mujeres en la fuerza laboral. El efecto sobre las mujeres que reciben cuido, que tienen una tasa de participación laboral de aproximadamente 66\%, ronda entre 7 y 14 puntos porcentuales. Los efectos sobre la participación son especialmente relevantes para las mujeres que no han terminado la secundaria. Todos estos resultados son estadísticamente significativos y robustos a diferentes especificaciones.

También se encuentra un aumento en las horas trabajadas semanales. El número de horas trabajadas de las mujeres ocupadas que utilizan cuido (que en promedio trabajan 36 horas por semana) es entre un $8 \%$ y $19 \%$ mayor que el de las mujeres con características similares que no utilizan cuido. Estos resultados fueron siempre positivos y en su mayoría estadísticamente significativos y robustos a diferentes especificaciones. Se encuentra, además, que el efecto sobre las horas trabajadas es substancialmente alto para las mujeres del primer quintil, aunque no siempre es estadísticamente significativo.

Una ampliación de los servicios de cuido en Costa Rica, además de contribuir con aspectos relacionados con la nutrición, desarrollo cognitivo y el crecimiento de los menores (Deutsch, 1998; Behrman, Cheng \& Todd, 2004; Berlinski \& Galiani, 2007; Nollenberger \& Perazzo, 2016), podría aumentar las posibilidades de las mujeres de participar tanto en el margen extensivo como intensivo en el mercado laboral. Esto significa que proveer estos servicios en lugares claves podría ayudar a sacar a muchos hogares de pobreza en la que actualmente viven. 
En la siguiente sección, se presentan los antecedentes. Luego, en la sección 3 se discuten los datos utilizados. En la sección, 4 se presenta la metodología. Los resultados se encuentran en la sección 5. Finalmente, presentamos nuestras conclusiones en la sección 6.

\section{ANTECEDENTES}

El comportamiento en la última década de la tasa de participación neta laboral femenina de Costa Rica resulta bastante irregular; sin embargo, la tasa se ha estabilizado en los últimos cuatro años alrededor del 51\%. Al comparar la tasa costarricense con respecto a la del mundo, se observa que se mantiene por debajo del promedio mundial en los últimos 4 años (ver gráfico 1).

La razón del estancamiento en la participación laboral femenina es multivariada; no obstante, al preguntarle a las mismas mujeres costarricenses la razón por la cual no se incorporarán a la fuerza laboral, el $63.3 \%$ de ellas responden que las obligaciones familiares en el hogar se lo impiden (Cálculo propio con datos del INEC, 2016; 2019).

El estancamiento se refleja más gravemente en el primer quintil de ingreso, donde la tasa de participación neta del $20 \%$ de las mujeres más pobres es de 30\%; mientras que las mujeres con el $20 \%$ de los ingresos más altos ronda el 70\%. El gráfico 2 muestra una mayor participación de las mujeres en el mercado laboral conforme aumenta el quintil de ingreso.

\section{Participar femenina en el mercado de trabajo}

Las teorías económicas más importantes para explicar las decisiones de las mujeres sobre si participan laboralmente se derivan de los modelos de oferta laboral. En estos modelos, los individuos tienen que tomar una decisión entre dos bienes: el ocio y el consumo de bienes los cuales se acceden a partir de las horas trabajadas que genera ingreso (Borjas, 1996; McConnel et al., 2003). Becker (1965), por otro lado, ve la decisión de distribución de tiempo en más actividades

\section{GRÁFICO 1}

\section{COSTA RICA Y EL MUNDO: PORCENTAJE DE MUJERES ENTRE 15-60 AÑOS QUE PARTICIPAN EN FUERZA DE TRABAJO, 2010-2019}

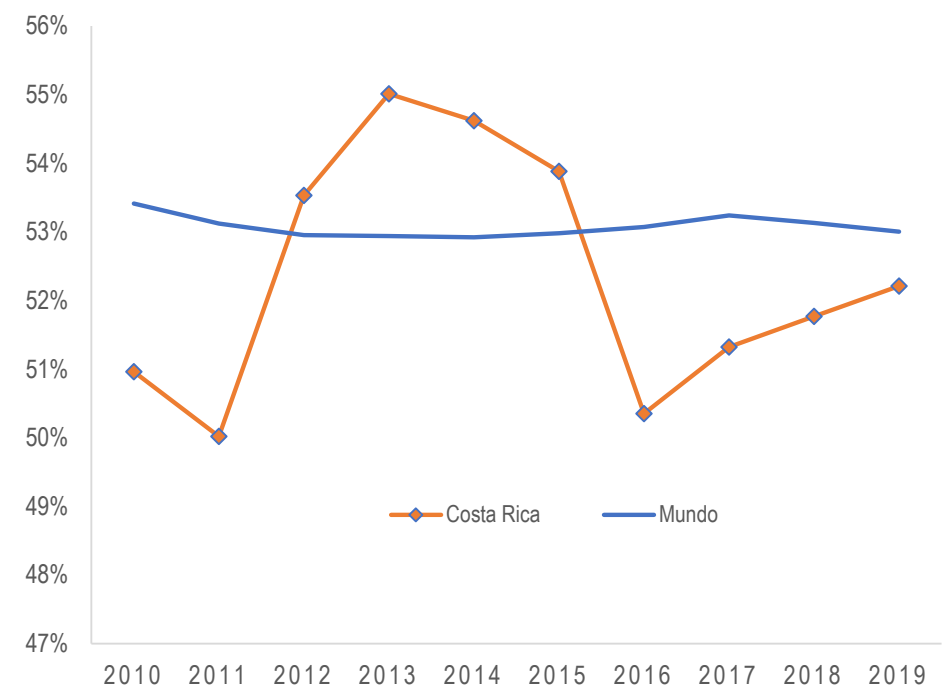

Fuente: Elaboración propia con datos del Banco Mundial (2020) 
que pueden ser vistas como insumos para el consumo de bienes. Dentro de este marco, el trabajo doméstico se puede ver como un insumo a la producción (Ferrada Bórquez \& Zarazosa Espina, 2010). Otro importante modelo económico considera que el proceso decisorio entre participar o no en el mercado de trabajo es consensuado dentro del hogar, así no solo se involucra a la mujer sino a la familia o el hogar como un todo (Ashenfelter \& Heckman, 1974).

Sin embargo, estas decisiones consensuadas o individuales se enmarcan dentro del contexto cultural presente en la sociedad (Gay, et al., 2018). El capital humano, por ejemplo, debido al costo de oportunidad, influye positivamente en que la mujer prefiera ingresar a participar en el mercado de trabajo (Domingo, 2011). También la estructura del hogar es un aspecto relevante. La presencia

GRÁFICO 2

\section{COSTA RICA: PORCENTAJE DE MUJERES ENTRE 15-59 AÑOS QUE PARTICIPAN EN LA} FUERZA DE TRABAJO SEGÚN QUINTILES DE INGRESO POR AÑO, 2011-2017

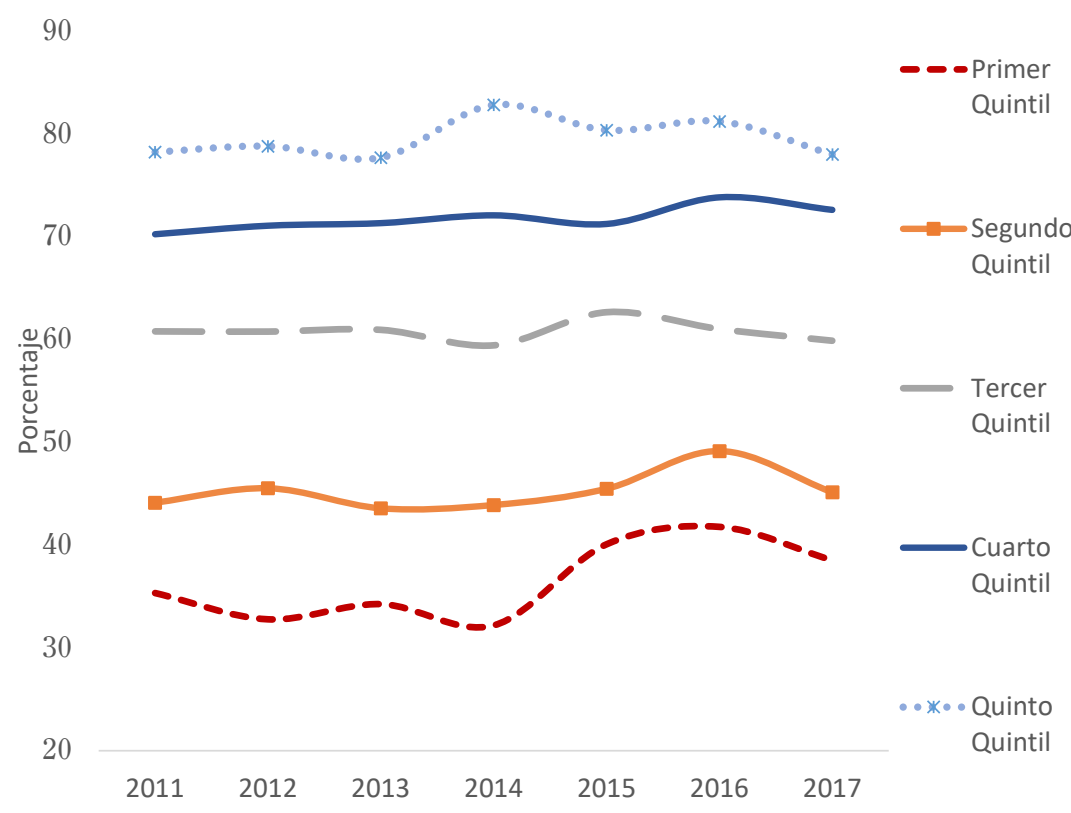

Fuente: Elaboración propia con datos de la ENAHO (INEC, 2011;2019).

de abuelas y adolescentes, entre otras, facilitan que la madre pueda trabajar fuera. Sin embargo, también la presencia de adultos proveedores y ancianos dificultaría la inserción de dichas mujeres en la fuerza laboral (Attanasio \& Vera-Hernández, 2004; Deutsch, 1998).

Los cambios en las leyes producidos por el órgano legislativo del país pueden también afectar la participación de las mujeres en el mercado laboral, afectando los costos y beneficios y modificando las relaciones culturales que existen. Específicamente para Costa Rica, la inclusión de la ley que obliga a los padres a dar una pensión alimenticia cuando no se hacen responsable de su manutención (Asamblea Legislativa de la República de Costa Rica, 1996), podría persuadir a la mujer de no participar laboralmente, al ya contar con esos ingresos (Ferrada Bórquez \& Zarzosa Espina, 2010). Sin embargo, también podría tener el efecto contrario sobre todo para aquellas mujeres en situación de pobreza, en línea con investigaciones que muestran que aumentos en los ingresos incentivan la participación laboral de las mujeres (Alzúa et al., 2013). Además, la ley de paternidad responsable tuvo un efecto negativo sobre la tasa de fertilidad (Ramos-Chaves, 2010). Estas reducciones en fertilidad podrían estar asociadas a aumentos en la participación laboral de las 
mujeres, como se encontró en Estados Unidos ante cambios legales a favor del uso de las pastillas anticonceptivas (Goldin et al., 2006).

Dado los resultados anteriores, es de esperar que reducciones en las demandas de tiempo en el hogar puedan tener un efecto positivo en la participación laboral de las mujeres. La evidencia empírica sobre la relación entre el cuido y la participación laboral en América Latina no es escaza, pero sus resultados han sido variados. Impactos positivos sobre la participación laboral se han encontrado en México (Ángeles et al., 2011), Ecuador (Rosero \& Oosterbeek, 2011), Colombia (Attanasio \& Vera, 2004), Argentina (Berlinsky \& Galiani, 2007), Alemania (Haan \& Wrohlich, 2009), Hungría (Lovász \& Szabo-Morvai, 2013) y Canadá (Baker et al., 2008). En casi todos esos estudios, se establece que hay una mayor incorporación de las madres en la fuerza laboral y un mayor número de horas trabajadas, que redunda en mayores salarios $y$, por ende, en mejores niveles de ingreso para el hogar.

No obstante, en Chile (Encina Galaz \& Martínez Alvear, 2009) y en Uruguay (Nollenberger \& Perazzo, 2016), encuentran que el impacto del cuido sobre la fuerza laboral es nulo. Entre las razones de la falta de efecto están la visión tradicional del rol de la mujer y la jornada de atención (Encina Galaz \& Martinez Alvear, 2009). Otra razón que puede limitar el efecto del cuido en la inserción laboral es que el mercado tenga una demanda limitada, especialmente para mujeres de baja educación (Nollenberger \& Perazzo, 2016). Otro efecto del cuido subsidiado es la posible liberación de ingreso en los hogares, lo que aumentaría el consumo de bienes, incluido el ocio que puede llevar a una reducción en la oferta laboral (Mateo Díaz \& Rodríguez-Chamussy, 2016).

En Costa Rica, aunque se ha argumentado que el cuido podría incentivar la participación laboral de las mujeres (Programa Estado de la Nación, 2015), todavía no existe evidencia cuantitativa suficiente que lo demuestre. De hecho, también se ha argumentado que ni RECUDI, ni el IMAS han tenido una verdadera estrategia para la incorporación de los padres y madres de los menores beneficiados a la participación laboral (Contraloría General de la República, 2015). Es por esto que es necesario analizar los impactos de este programa sobre la oferta laboral de las mujeres en Costa Rica.

\section{Política de cuido infantil en Costa Rica}

Desde el siglo XX, el Gobierno costarricense comenzó a crear diferentes instituciones proveedoras de cuido infantil. La primera fue el Patronato Nacional de la Infancia en 1930, con un programa enfocado en el "rescate" de menores en riesgo social y vulnerabilidad. En 1971, se creó el IMAS, pero no fue hasta 1992 que esta institución empezó a subsidiar de manera organizada el cuido infantil, a través del programa Hogares Comunitarios (HC). Por último, en 1973 se crean los Centros de Educación y Nutrición y Centros Infantiles de Atención Integral (CEN-CINAI), con un enfoque en nutrición, promoción del crecimiento y desarrollo de los menores (Fondo de Desarrollo Social y Asignaciones Familiares [FODESAF], 2017).

Hasta el 2010, la cobertura pública y mixta de los servicios de cuido y atención integral para la primera infancia en el país se consideraba de baja magnitud. En efecto, la cobertura de menores de hasta 7 años con nivel de pobreza alcanzó apenas el 11,5\% para ese año, incluyendo los servicios ${ }^{1}$ del CEN-CINAI, PANI y del IMAS (Contraloría General de la República, 2011). Por este motivo, la Contraloría General de la República instó a las autoridades a aumentar la cantidad de niños que iban a ser beneficiados con el cuido, sobre todo ante la alta demanda de familias en condición de pobreza que podrían necesitar el acceso a dichos servicios (Contraloría General de la República, 2011).

\footnotetext{
1 Los programas de cuido infantil del PANI, CEN-CINAI y del IMAS pertenecen a la Redcudi y son los que proveen servicios directamente o a través de subsidios a menores.
} 
Ante esto, a través de la Ley 9220, se crea La Red Nacional de Cuido y Desarrollo Infantil (REDCUDI) en al año 2014, confirmando el decreto del ejecutivo firmado desde el 2010. Su objetivo es articular las actividades que se desarrollan en el país en materia de cuido y desarrollo Infantil (IMAS, 2020). Como parte de la ley, se crea un programa que maneja el IMAS que subsidia la inscripción de menores en diferentes alternativas de cuido privada. Las tres alternativas que más reciben menores son: los $\mathrm{HC}$, CECUDI y los Centros Infantiles de Atención Integral no Gubernamentales (Universidad de Costa Rica-CICAP-ESP, 2016).

El Programa de Microempresas para la Atención Infantil-Hogares Comunitarios (HC) se crea mediante el Decreto Ejecutivo No21391-TSS-MEP-S del año 1992 que antecede la creación de REDCUDI. Constituye el primer programa de cuido infantil, con la participación del sector público y privado, $y$ con sujeción a las políticas de Bienestar Social del Gobierno de la República (FODESAF, 2017). Específicamente, los niños y niñas reciben en los HC cuido y atención en sus necesidades básicas, sin estimulación temprana (Grillo et al., 2010).

La Cobertura temporal y geográfica de los Hogares Comunitarios ha ido disminuyendo. Así, en noviembre de 1993, existían $700 \mathrm{HC}$, que atendían 5000 niñas y niños (Organización de Estado Iberoamericanos [OEI], 2018), pero para finales del 2017, se reportaron únicamente 178 centros, que albergan 1.916 menores (IMAS, 2018). La principal razón de esta disminución en cobertura es la falta de apoyo gubernamental, percibida desde mediados de la década anterior (Cañas Calvo et al., 2007).

Los CECUDI surgen a partir del año 2012, por medio del Decreto No. 36916, en ellos se da atención integral a la población infantil de manera interdisciplinaria (FODESAF, 2017). Corresponden a una alianza estratégica entre el IMAS, FODESAF y las municipalidades. Pueden ser administrados desde los gobiernos municipales o por diferentes iniciativas privadas. La cobertura de los CECUDI muestra un fuerte aumento, aunque según la Contraloría General de la República (2015), ha existido una falta de planificación en la inversión de los nuevos CECUDI. Para el 2013, funcionaban 16, que atendían 1.021 infantes. En el 2018 hay 66 centros, distribuidos en las 7 provincias, principalmente ubicados en la mayoría de los cantones más urbanos del país donde se cuidan a 5.093 menores. Esto significa que se atiende un promedio de 69 niños y niñas por centro (IMAS, 2018).

Además de estas dos alternativas, también existen los Centros Infantiles de Atención Integral no Gubernamentales, son parte de la Redcudi. Estos centros privados son los que más atienden menores subsidiados por el IMAS. En el 2016, un 70\% de los subsidios a menores fueron atendidos por ellos (IMAS, 2017).

La Redcudi tiene un servicio de hasta 12 horas diarias y atiende a infantes de 0-12 años (FODESAF, 2018). Esto depende del centro de atención. El IMAS, en 2019, pagaba mensualmente por cada menor atendido $\$ 131.000$ en los CECUDI (Chinchilla Cerdas, 2019) y en el 2017, $\$ 88.000$ en los HC (Chinchilla Cerdas, 2017); con fondos provenientes de FODESAF.

\section{El proceso de selección y retos del programa de subsidios}

Para recibir el subsidio, las familias deben llevar a cabo un proceso de solicitud, donde se verifica si la familia beneficiada cumple con una serie de requisitos. Primero (FODESAF, 2018), debe solicitar una cita en el IMAS, donde se recolecta información sobre su estatus económico ${ }^{2}$. Además, se debe entregar certificados emitidos por el centro de cuido que aseguran la disponibilidad de un espacio. También se solicitan documentos que demuestren los ingresos de cada uno de

2 Para fijar la condición socioeconómica y priorizar la atención de los hogares, el IMAS utiliza el modelo estadístico del puntaje, llamado Sistema de Información de la Población Objetivo (SIPO). Este sistema se obtiene a partir del análisis de la información de la ENAHO y se complementa con la Ficha de Información Social (FIS), que es completada por el solicitante del beneficio. Las familias que no ameriten recibir el beneficio, que sean descubiertas por los controles y mecanismos utilizados por la institución en el seguimiento, serán excluidas). 
los miembros del núcleo familiar (pueden ser declaraciones juradas). Luego, el IMAS analiza la solicitud. Una vez aprobada la solicitud, el beneficiario podrá hacer uso del centro y los fondos se transferirán directamente del IMAS hacia el centro de cuido.

Se ha argumentado que el requisito de estar en una situación económica vulnerable puede también incidir en la participación de la mujer en el mercado laboral. Esto debido a que obliga al IMAS suspender el beneficio de subsidio si se produce una mejora económica en el hogar que supere el límite establecido para ser beneficiario (FODESAF, 2018) que puede resultar a consecuencia de la inserción al mercado laboral. Esto resulta un contrasentido, pues podría provocar que la mujer desista de entrar al mercado laboral, especialmente al formal, para que el ingreso del hogar no supere ese límite.

La solución a esto no parece sencilla, debido a que esta discusión va de la mano con los problemas comunes de filtraciones en los programas sociales, que dicha reglamentación pretende evitar. Aun con esta normativa, en sendas evaluaciones realizadas a la Redcudi se llegan a resultados contradictorios. Mientras para el estudio de la Universidad de Costa Rica-CICAP-ESP (2016) el subsidio es otorgado sin filtraciones, pues los niños y niñas incluidos dentro del proceso pertenecen efectivamente a los sectores que se necesita abordar como parte de la política social del país. También existe la evaluación del Ministerio de Trabajo y Seguridad Social (2016) que indica que la falta de documentación de respaldo de la condición socioeconómica en los expedientes de los beneficiarios del IMAS podría materializar el riesgo de filtraciones.

Otro reto es la calidad del servicio de cuido. Si las madres perciben que el servicio es bajo en calidad-confianza, lo usarían menos (Mateo Díaz \& Rodríguez-Chamussy, 2016). En contraste, si la atención es percibida como buena en calidad-confianza, podría hacer que las mujeres que en la actualidad están trabajando y que contratan servicios privados de cuido se trasladen a estas alternativas, lo que tampoco aumentaría la oferta de trabajo. Sin embargo, la Universidad de Costa Rica-CICAP-ESP (2016) aplicó una evaluación, donde se concluye que el cuido de las dos alternativas evaluadas, HC y CECUDI, en la presente investigación es de calidad. Según la opinión dada por las encargadas de los menores, el servicio es calificado como de muy buena calidad y confianza. Los usuarios también califican de forma positiva la infraestructura y el equipamiento de los centros.

Además, no existe ningún condicionamiento de participar laboralmente para acceder al subsidio (FODESAF, 2017). Un caso contrario al costarricense sucede por ejemplo en Chile y Alemania, donde es obligatorio para la madre participar laboralmente para poder disfrutar del servicio (Encina Galaz \& Martínez Alvear, 2009; Haan \& Wrohlich, 2009). Esta falta de condicionamiento no contribuye a la inserción laboral de la mujer.

Las carencias encontradas en la Redcudi hacen que los estudios de los programas individuales, en especial, las evaluaciones de impacto cobren aún más importancia. Si se demuestran los impactos esperados en las mujeres de los hogares beneficiarios, la articulación de la política puede amplificar esos resultados, lo mismo si los programas requieren modificaciones. En síntesis, las evaluaciones como la hecha en esta investigación cobran cada vez más relevancia, porque orientan las decisiones políticas en esta materia.

\section{DATOS}

Los datos utilizados en esta investigación fueron tomados de la ENAHO (INEC 2016; 2019) ${ }^{3}$. Dicha encuesta contiene información para una gran cantidad de variables que muestran las características de los hogares y la población en situación de pobreza costarricense. Su información

3 Antes de estos años, en a ENAHO no hay información sobre los programas de cuido del IMAS. 
se presenta a nivel de individuos ${ }^{4}$ y de hogares, dentro de 1.136 unidades primarias de muestreo (UPM) ${ }^{5}$. La unidad de observación corresponde a mujeres entre los 18 y 43 años, de la zona urbana y con educación primaria, secundaria o universitaria de pregrado y grado. Dichas mujeres pertenecen a un hogar donde hay menores de 13 años (hogar con o sin beneficio de cuido) y a los 3 quintiles más bajos de ingreso. Para efectos del análisis, se excluyen de la muestra aquellas mujeres que pertenezcan a hogares beneficiados con los programas CEN-CINAI y otros tipos de subsidio del IMAS, dado que los hogares de estas mujeres reciben subsidios que son similares a los que reciben los del cuido, lo que podría contaminar la estimación del impacto.

Inicialmente, se cuenta con 38.648 encuestadas de hogares donde al menos vive una persona menor de 13 años. De estas, 17.250 están entre los 18-43 años y 16.558 no reciben ninguna otra ayuda del IMAS o CEN-CINAI. De ese subgrupo, 12.705 pertenecen a los tres quintiles más pobres de la población. Además, se eliminan observaciones de las de zonas rurales, debido a su escasez en esta zona. Esto nos deja 8.005 observaciones en zonas urbanas. Dentro del grupo de mujeres no beneficiadas, solamente se incluyen aquellas que vivan en las mismas UPM de las que sí han recibido el beneficio. Esta última restricción nos deja con 2355 mujeres. También, se descartan las que tienen educación superior completa, ya que en la muestra no había ninguna tratada con esta condición. Finalmente, se excluyen mujeres sin ningún tipo de educación y con educación técnica incompleta, porque solo había tres observaciones tratadas en la muestra con esas condiciones ${ }^{6}$. Esto nos deja con 2.275, de las cuales 224 pertenecen a la población intervenida y el resto sirven de comparación (ver gráfico 3).

\section{GRÁFICO 3 \\ COSTA RICA: NÚMERO DE OBSERVACIONES ELEGIBLES Y DESCRIPCIÓN DEL PROCESO DE SELECCIÓN MUESTRAL, 2016-2019}

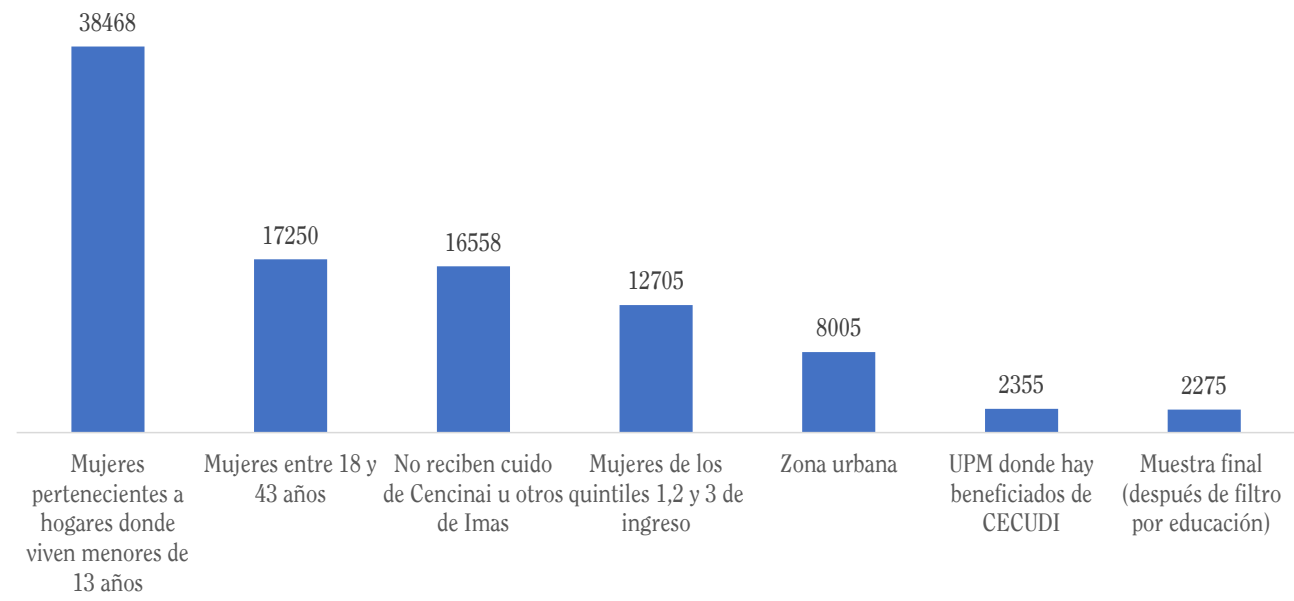

Fuente: Elaboración propia con datos de la ENAHO (INEC, 2011;2019).

\footnotetext{
4 La muestra inicial la componen 141,808 individuos.

5 Son áreas geográficas en las que se divide cada distrito del país y que contienen en promedio 150 viviendas en las zonas urbanas y 100 viviendas en promedio en las zonas rurales.

6 Los niveles de educación que quedan en la muestra para que esta quede lo más similar posible son: Quedan en la muestrea solo las mujeres que tienen: primaria incompleta, primaria completa, secundaria académica incompleta, secundaria académica completa, secundaria técnica completa, educación superior de pregrado y grado.
} 


\section{CUADRO 1 \\ COSTA RICA: ESTADÍSTICAS DESCRIPTIVAS DE LAS MUJERES SELECCIONADAS PARA LA EVALUACIÓN DE IMPACTO SEGÚN LAS VARIABLES DEPENDIENTES Y LA INDEPENDIENTE DE INTERÉS}

\begin{tabular}{|c|c|c|c|c|}
\hline Variables & Todas & Con cuido & Sin Cuido & Diferencia (2) - (3) \\
\hline & (1) & (2) & (3) & $(4)$ \\
\hline Participación laboral & $57,23 \%$ & $66,07 \%$ & $56,26 \%$ & $9,81^{* * *}$ \\
\hline Horas normales+ semanalmente & 34.77 & 36.20 & 34.59 & 1.61 \\
\hline Cuido++ & $12,95 \%$ & $100 \%$ & $0 \%$ & $100 \%$ \\
\hline \multicolumn{5}{|l|}{ Características Individuales } \\
\hline Edad (años) & 29.95 & 28.72 & 30.09 & $-1.37 * * *$ \\
\hline Tiene pareja (dicotómica) & $48.99 \%$ & $40,00 \%$ & $49,97 \%$ & $-9,97 * * *$ \\
\hline \multicolumn{5}{|l|}{ Educación (fracciones): } \\
\hline Primaria o menos & $31,56 \%$ & $35,27 \%$ & $31,11 \%$ & 4,16 \\
\hline Secundaria Incompleta & $34,90 \%$ & $32,14 \%$ & $35,20 \%$ & $-3,06$ \\
\hline Secundaria completa & $24,04 \%$ & $25,00 \%$ & $23,94 \%$ & 1,06 \\
\hline Superior de pregrado y grado & $9,54 \%$ & $7,59 \%$ & $9,75 \%$ & $-2,16$ \\
\hline Es jefa de hogar & $27,02 \%$ & $38,67 \%$ & $25,74 \%$ & $12,92^{* * *}$ \\
\hline Inmigrante $^{1}$ & $45,70 \%$ & $52,90 \%$ & $44,90 \%$ & $7,94 \% * * *$ \\
\hline Pensión alimenticia & $16,70 \%$ & $23,60 \%$ & $16,00 \%$ & $16,60 \% * *$ \\
\hline \multicolumn{5}{|l|}{ Características del hogar } \\
\hline Región Central & $71,00 \%$ & $72,44 \%$ & $70,84 \%$ & 1,60 \\
\hline Casa propia & $47,14 \%$ & $39,55 \%$ & $47,98 \%$ & $-8,42 * *$ \\
\hline Niños menores de 6 años & 0.54 & 0.84 & 0.51 & $0.32^{* * * *}$ \\
\hline Niñas menores de 6 años & 0.44 & 0.74 & 0.41 & $0.33^{* * * *}$ \\
\hline Niños entre 7 y 12 años & 0.44 & 0.39 & 0.44 & -0.05 \\
\hline Niñas entre 7 y 12 años & 0.38 & 0.34 & 0.38 & -0.04 \\
\hline Niños entre 13 y 17 años & 0.20 & 0.22 & 0.20 & 0.02 \\
\hline Niñas entre 13 y 17 años & 0.22 & 0.19 & 0.23 & -0.03 \\
\hline Hombres entre 18 y 65 años & 1.02 & 0.95 & 1.02 & -0.07 \\
\hline Mujeres entre 18 y 65 años & 1.76 & 1.84 & 1.76 & 0.08 \\
\hline Hombres mayores de 66 años & 0.06 & 0.04 & 0.06 & -0.02 \\
\hline Mujeres mayores de 66 años & 0.07 & 0.08 & 0.07 & 0.01 \\
\hline Quintil de ingreso 1 & $34,92 \%$ & $46,66 \%$ & $33,64 \%$ & $13,02^{* * *}$ \\
\hline Quintil de ingreso 2 & $35,98 \%$ & $29,78 \%$ & $36,66 \%$ & $-6,88^{* *}$ \\
\hline Quintil de ingreso 3 & $29,08 \%$ & $23,56 \%$ & $29,69 \%$ & $-6,14^{*}$ \\
\hline Hogar jefeado por mujer & $51,58 \%$ & $72,44 \%$ & $49,29 \%$ & $23,15 \% * * *$ \\
\hline Observaciones & 2275 & 224 & 2051 & 1827 \\
\hline
\end{tabular}

Significancia: $* 10 \% * * 5 \% * * * 1 \%$

1 nació en otro país o en otro cantón del que nació en Costa Rica.

Fuente: Elaboración propia con datos de la ENAHO (INEC, 2011;2019). 
Las variables dependientes que se utilizan en esta investigación se pueden observar en el cuadro 1 y son las siguientes:

- Participa en la fuerza laboral: es una variable dicotómica; toma el valor de 1 si participa y 0 si no. Las mujeres que participan son las que están empleadas o las que buscan empleo.

- Horas normales trabajadas semanalmente: Es una variable de cuenta, ya que corresponde al número de horas trabajadas.

La variable independiente de interés es la participación en el programa y se le denomina "Cuido". Es una variable dicotómica que toma el valor de 1 cuando la mujer pertenece a un hogar que recibe cuido por parte del IMAS y 0 cuando no recibe. Debido a la partición que se hizo, solo se consideran mujeres en hogares donde se utilizó HC y CECUDI o donde no se utilizó ningún tipo de cuido.

Además, se utilizan otras variables identificadas en la literatura como factores explicativos de la participación en la fuerza de trabajo y que, además, puedan estar correlacionadas con la utilización del cuido. Estas variables se describen en el cuadro 1.

El primer grupo de variables corresponden a características individuales o propias de la mujer. El segundo describe las características del hogar donde residen.

En el cuadro 1, también se presentan los promedios de cada una de las variables dependientes y las independientes, para la muestra total (columna 1), para el grupo intervenido, mujeres que residen en hogares cuyos menores de 13 años participan en el programa (columna 2), y para las mujeres sin cuido, mujeres residentes en hogares no beneficiarios (columna 3).

Al comparar las medias de las variables dependientes (columna 4), la diferencia en las tasas de participación laboral entre los dos grupos es altamente significativa. Esta diferencia es de 9,81 p.p. a favor de la población intervenida. En cuanto a las horas normales trabajadas semanalmente, no se encuentran diferencias significativas. Sin embargo, no es adecuado hacer conclusiones causales a partir de estas diferencias. Esto debido a que en la columna 4 se muestra que los hogares de las mujeres intervenidas son diferentes a las mujeres no intervenidas en edad, tenencia de pareja, prevalencia de jefatura femenina, tenencia de casa propia, presencia de niños menores a 6 años, y pertenencia al primer quintil de ingreso. Todas estas son variables que pueden afectar la inserción en el mercado laboral independientemente del uso de cuido.

\section{METODOLOGÍA}

Para poder estimar el efecto causal del cuido se requiere calcular en forma adecuada lo que habría ocurrido con las mujeres de los hogares beneficiados, si no lo habrían sido. Este escenario, conocido como contra-fáctico, para la misma persona en el mismo momento no es observable. Solo se puede estimar utilizando observaciones mientras no hayan sido tratadas. Sin embargo, es difícil poder asegurar que las observaciones que no han recibido el cuido habrían tenido el mismo resultado que las observaciones que lo recibieron si no lo habrían recibido. Esta diferencia se le conoce como sesgo (Angrist \& Piscke, 2008). El sesgo muchas veces es generado por el proceso de autoselección de las mujeres en un grupo versus el otro.

El reto metodológico en esta investigación consiste en eliminar este sesgo. Para resolver este reto, se hacen comparaciones entre aquellas mujeres con y sin cuido que tienen características similares (condicionamiento). En este artículo utilizamos dos estrategias de condicionamiento: emparejamiento (matching) y regresión. Las variables que se utilizan para el condicionamiento son las características individuales y las características del hogar presentadas en el cuadro 1. 
Las técnicas de emparejamiento son ampliamente utilizadas en evaluación de impacto (Kam \& Palmer, 2008; Mayer, 2011; Goraus \& Tyrowicz, 2014). En este artículo, específicamente se utiliza como estrategia de emparejamiento: propensity score matching (PSM). El cálculo por medio del PSM se logra en 2 etapas. En la primera etapa, se utiliza un modelo probit para estimar con las características observables de cada mujer (variables de condicionamiento), la probabilidad de haber recibido el beneficio del cuido.

Una vez que se estima la probabilidad para cada mujer, se realiza un proceso de emparejamiento para cada una de las observaciones tratadas con la observación no tratada con la probabilidad más cercana. Con esto, se busca conseguir un grupo de control con probabilidad similar de recibir el tratamiento, dadas las variables observadas (Dehejia \& Wahba, 2002). Se empareja cada observación tratada con uno o dos vecinos más cercanos. Complementariamente, se utiliza un caliper de $0,01^{7}$. Además, el emparejamiento se hace con reemplazo, donde cada unidad tratada puede ser comparada con la unidad de control más cercana; aunque esta unidad de control ya haya sido pareada con otra unidad tratada. Con este procedimiento, se construyen submuestras de observaciones tratadas y control similares.

Sin embargo, diferencias entre el grupo tratado y grupo control pueden permanecer después del emparejamiento. Para eliminar los posibles sesgos remanentes que estas diferencias pueden generar, se estiman los efectos con mínimos cuadrados ordinarios (MCO), modelos probabilísticos (logit, binomial o multinomial) o modelos con truncamiento (tobit) controlando por las variables control.

Sin embargo, todavía podrían existir características no observables que hagan que los grupos de comparación sean diferentes, inclusive luego de implementar los pasos anteriormente descritos. Se intentó resolver este otro reto, parcialmente, con dos estrategias. La primera es utilizar solamente observaciones no tratadas que pertenecen a la mínima unidad geográfica (UPM) de las mujeres beneficiadas. Existen varias características no observables que están correlacionadas con el lugar de residencia. Por esto, al enfocar el estudio en observaciones del mismo lugar de proveniencia, varias características no observables serán balanceadas.

Una segunda estrategia utilizada para eliminar diferencias no observadas es controlar dentro de la etapa de regresión con variables de cohortes. Las variables de cohortes son variables dicotómicas que capturan el efecto por grupos de individuos de la misma edad. En cada etapa de la vida las personas comparten experiencias $y$, por lo tanto, grupos de individuos que pasan por esas etapas de la vida al mismo tiempo generan características similares. De esta manera, se logra aproximar los resultados a los que se obtendrían si se contara con un panel de datos (Deaton, 1985).

En resumen, la especificación que se utiliza con las submuestras emparejadas es:

$$
Y_{i}=\beta_{1}+\beta_{2} T_{i}+\sum_{k=3}^{n} \beta_{k} A_{k i}+\sum_{j=n+1}^{n} \beta_{j} G_{j i}+\mu_{i}
$$

donde:

$Y_{i}=$ variable dependiente: si la mujer participa laboralmente o no; horas normales trabajadas semanalmente;

$T_{i}=$ variable dicotómica para el tratamiento: si la mujer pertenece o no a un hogar que recibe cuido infantil;

$A_{k i}=k$ variables de control $y$;

$G_{j i}=j$ cohortes de edad;

7 Al realizar la prueba de balance, con la nueva muestra resultante después del emparejamiento, se encontró que prácticamente para todas las variables explicativas la diferencia entre las medias del grupo de control (sin cuido) y el grupo de intervenidas (con cuido) se redujeron y no son significativas. Por lo que a través del matching se logra que los dos grupos sean muy similares. 
El coeficiente $\beta_{2}$ captura el efecto del tratamiento. Se esperaría que no esté sesgado por diferencias observables que puedan existir entre las variables que determinan el tratamiento y la participación (debido a que se utilizan para controlar en la regresión y el pareo); ni por factores que no se observan que generan diferencias sobre el cuido entre generaciones (ya que se controla por cohortes; Lang et al., 2015), ni mediante las diferencias geográficas (por la selección de la muestra).

Con la especificación presentada, el supuesto de identificación es que no existen factores no observables que estén relacionados con el cuido y con las decisiones del mercado laboral para mujeres que tengan edad similar y que viven en la misma unidad geográfica. Si este supuesto no se cumple, la estimación del efecto podría estar sesgado. Esto podría ocurrir si es que dos mujeres de la misma edad, que viven en el mismo lugar, y que tienen variables observables similares, tenga una característica no observable diferente que hagan que una decida, por ejemplo, trabajar y utilizar cuido y que la otra no. Con esto, se está asumiendo implícitamente que luego de controlar por todos esos factores, las razones por las cuales se decide ser beneficiario del cuido son aleatorias. La única forma para poder realmente asegurarse de esto es utilizar una estrategia experimental y este es un estudio observacional.

Sin embargo, diferentes especificaciones se llevarán a cabo para asegurarse que las consecuencias sobre los resultados son reducidas.

\section{RESULTADOS}

El cuadro 2 muestra los efectos del cuido sobre la participación en la fuerza laboral utilizando cinco especificaciones. En las primeras 4 columnas, se utilizan modelos lineales. En la columna 1 , se examina el efecto comparando, sin ninguna variable control, la participación entre mujeres con el beneficio de cuido y sin este; en la columna 2, se incorporan los controles correspondientes a las características de la mujer; posteriormente, en la columna 3, se incluyen las características del hogar; finalmente, en la columna 4, se añade el conjunto de cohortes de edad. En la columna 5 , se muestran los resultados del efecto marginal de un modelo logit binomial utilizando todos los controles previamente descritos. Además, para cada especificación antes descrita, se presentan los resultados utilizando la muestra seleccionada en la que previamente se ha buscado tener un grupo control muy similar al grupo de mujeres intervenidas. En la segunda y tercera fila se utiliza propensity score matching con un caliper de 0,01, con el propósito de filtrar aún más la muestra y emparejar a las mujeres realmente más similares. Así, en la segunda fila se utiliza PSM con el vecino más cercano y en la tercera fila se utiliza PSM con los dos vecinos más cercanos. 


\section{CUADRO 2}

\section{COSTA RICA: ESTIMACIÓN DEL IMPACTO DEL CUIDO SOBRE LA PARTICIPACIÓN EN LA FUERZA LABORAL DE LAS MUJERES DE 18 A 43 AÑOS}

\begin{tabular}{|c|c|c|c|c|c|}
\hline \multirow{2}{*}{ Muestras } & \multicolumn{4}{|c|}{ Modelos usando Mínimos Cuadrados Ordinarios } & \multirow{2}{*}{$\frac{5 \text { Logit }}{5}$} \\
\hline & 1 & 2 & 3 & 4 & \\
\hline Toda la muestra & $0.099 * * *$ & $0.086^{* * *}$ & $0.089^{* * * *}$ & $0.090^{* * * *}$ & $0.093^{* * *}$ \\
\hline$(\mathrm{n}=2275)$ & $(0.034)$ & $(0.033)$ & $(0.033)$ & $(0.033)$ & $(0.034)$ \\
\hline Pareo $[\mathrm{n}=1$ y cal. 0,01$]$ & 0.071 & $0.083^{*}$ & $0.100^{* *}$ & $0.106^{* *}$ & $0.108^{* *}$ \\
\hline$(\mathrm{n}=408)$ & $(0.051)$ & $(0.049)$ & $(0.048)$ & $(0.047)$ & $(0.045)$ \\
\hline Pareo $[\mathrm{n}=2$ y cal. 0,01$]$ & $0.110^{* *}$ & $0.125^{* * *}$ & $0.141^{* * *}$ & $0.139^{* * *}$ & $0.140^{* * *}$ \\
\hline$(\mathrm{n}=561)$ & $(0.044)$ & $(0.043)$ & $(0.041)$ & $(0.041)$ & $(0.039)$ \\
\hline \multicolumn{6}{|l|}{ Controles } \\
\hline Características de la mujer & No & Sí & Sí & Sí & Sí \\
\hline Características del hogar & No & No & Sí & Sí & Sí \\
\hline Cohortes & No & No & No & Sí & Sí \\
\hline
\end{tabular}

Significancia: $* 10 \% * * 5 \% * * * 1 \%$

Fuente: Elaboración propia con datos de la ENAHO (INEC, 2011;2019).

Se observa que todos los coeficientes estimados son positivos y rondan entre los 7 y 14 p.p.. Además, todas las especificaciones, excepto una, son estadísticamente significativas. Las especificaciones 4 y 5 , que son las que tienen todos los controles, muestran resultados entre 9 y 14 p.p. $y$ todos los coeficientes son estadísticamente significativos. A partir de estas dos últimas dos especificaciones, concluimos que el cuido de los HC y CECUDI tienen un impacto positivo en el margen extensivo de la oferta laboral de las mujeres de los hogares beneficiados.

En los hogares de los tres quintiles más pobres, la tasa de participación es menor al 55\% (ver gráfico 2). Por lo tanto, los aumentos que podrían estar alrededor de 10 puntos porcentuales son sumamente importantes. Nuestra muestra tiene características diferentes, a pesar de que pertenece a los tres quintiles más pobres de la población. Sin embargo, su tasa de participación promedio es del $57 \%$ (ver cuadro 1), que es también relativamente baja.

El cuadro 3 muestra los resultados para la variable del logaritmo de las horas normales trabajadas semanalmente. Las estimaciones de los coeficientes con el modelo Tobit y MCO muestran valores positivos y económicamente relevantes. Todos los resultados del cuadro muestran resultados positivos que rondan entre el $8 \%$ y el 19\%. Sin embargo, al tomar en cuenta toda la muestra, no existe diferencia estadísticamente significativa entre los dos grupos. En el caso de las muestras después de los emparejamientos, tener el beneficio del cuido aumenta la probabilidad de que la mujer trabaje más horas semanalmente en más de 19 por ciento en forma estadísticamente significativa. 


\section{CUADRO 3 \\ COSTA RICA: ESTIMACIÓN DEL IMPACTO DEL CUIDO SOBRE EL LOGARITMO DE LAS HORAS TRABAJADAS NORMALES SEMANALES DE LAS MUJERES DE 18 A 43 AÑOS (SE UTILIZAN TODAS LAS OTRAS VARIABLES DE CONTROL)}

\begin{tabular}{lrr}
\hline \multicolumn{1}{c}{ Muestras } & Tobit & \multicolumn{1}{c}{ MCO } \\
\hline Toda la muestra $(\mathrm{n}=992)$ & 0.0811 & 0.0802 \\
& $(0.0793)$ & $(0.0802)$ \\
Pareo [n=1 y cal. 0,01] & $0.2020^{*}$ & $0.1980^{*}$ \\
(n=212) & $(0.1120)$ & $(0.1190)$ \\
& & \\
Pareo [n=2 y cal. 0,01] & $0.1930 * *$ & $0.1900 *$ \\
$(\mathrm{n}=287)$ & $(0.0944)$ & $(0.0992)$
\end{tabular}

Significancia: $* 10 \% * * 5 \% * * * 1 \%$. Se utilizan como variables control, las características de la mujer, del hogar y cohortes.

Fuente: Elaboración propia con datos del INEC, 2016;2019.

Los resultados permiten concluir que el cuido contribuye en la incorporación de más mujeres al mercado laboral y parece influir en forma positiva en la magnitud de horas sobre aquellas mujeres que ya están laborando. Resultados similares a este se obtuvieron en México (Ángeles et al., 2011), Ecuador (Rosero \& Oosterbeek, 2011) y Colombia (Attanasio \& Vera-Hernández, 2004).

Finalmente, cabe destacar que los valores obtenidos no cambian cualitativamente al realizar variaciones en los métodos econométricos. Tampoco cambian ante cambios en las muestras utilizadas para comparar. Por lo tanto, podemos concluir que los resultados son robustos.

Además, en el cuadro 4 se exploró si las características de las mujeres podrían hacer que los efectos de la política variarían. Estas estimaciones se realizaron sin utilizar pareo, debido a que el número de observaciones cae substancialmente. El efecto del cuido sobre la participación es más fuerte para el quintil 2 que para el quintil 1 y 3. También, este efecto es más fuerte para las mujeres que no han completado la secundaria. 


\section{CUADRO 4 \\ COSTA RICA: ESTIMACIÓN DEL IMPACTO DEL CUIDO SOBRE LA PARTICIPACIÓN LABORAL Y EL LOGARITMO DE LAS HORAS TRABAJADAS NORMALES SEMANALES DE LAS MUJERES DE 18 A 43 AÑOS POR QUINTIL Y NIVEL DE EDUCACIÓN, UTILIZANDO TODA LA MUESTRA}

\begin{tabular}{|c|c|c|c|c|}
\hline \multirow{2}{*}{ Muestras } & \multicolumn{2}{|c|}{ Participación } & \multicolumn{2}{|c|}{ Horas trabajadas } \\
\hline & Logit & $\mathrm{MCO}$ & Tobit & $\mathrm{MCO}$ \\
\hline \multicolumn{5}{|l|}{ Quintil 1 } \\
\hline Efecto & 0.074 & 0.073 & $0.287^{*}$ & 0.279 \\
\hline Error estándar & $(0.050)$ & $(0.055)$ & $(0.168)$ & $(0.180)$ \\
\hline Observaciones & 795 & 795 & 230 & 230 \\
\hline \multicolumn{5}{|l|}{ Quintil 2} \\
\hline Efecto & $0.137^{* *}$ & $0.126^{* *}$ & 0.015 & 0.015 \\
\hline Error estándar & $(0.062)$ & $(0.060)$ & $(0.130)$ & $(0.136)$ \\
\hline Observaciones & 818 & 818 & 354 & 354 \\
\hline \multicolumn{5}{|l|}{ Quintil 3} \\
\hline Efecto & 0.069 & 0.072 & 0.099 & 0.099 \\
\hline Error estándar & $(0.067)$ & $(0.066)$ & $(0.097)$ & $(0.101)$ \\
\hline Observaciones & 662 & 662 & 408 & 408 \\
\hline \multicolumn{5}{|c|}{ Secundaria incompleta o menos } \\
\hline Efecto & $0.125^{* * *}$ & $0.119 * * *$ & 0.062 & 0.061 \\
\hline Error estándar & $(0.042)$ & $(0.041)$ & $(0.102)$ & $(0.104)$ \\
\hline Observaciones & 1511 & 1511 & 602 & 602 \\
\hline \multicolumn{5}{|c|}{ Secundaria completa o más } \\
\hline Efecto & 0.042 & 0.037 & 0.056 & 0.056 \\
\hline Error estándar & $(0.058)$ & $(0.058)$ & $(0.126)$ & $(0.131)$ \\
\hline Observaciones & 764 & 764 & 390 & 390 \\
\hline
\end{tabular}

Significancia: $* 10 \% * * 5 \% * * * 1 \%$. Se utilizan como variables control, las características de la mujer, del hogar y cohortes.

Fuente: Elaboración propia con datos del INEC, 2016;2019.

El efecto del cuido sobre las horas trabajadas crece substancialmente en magnitud para el quintil más pobre (más de 3 veces más) si se compara con el estimador mostrado en el cuadro 3, cuando se utilizó toda la muestra. Además, se hace estadísticamente significativo utilizando el modelo Tobit. Un resultado similar en magnitud se encuentra con MCO, aunque no es estadísticamente significativo. Se puede ver que la falta de significancia probablemente se debe al reducido número de observaciones. Una de las posibles explicaciones de estos resultados es que el quintil más pobre tiene más necesidades de cuido que el resto de quintiles analizados y, simultáneamente, una mayor necesidad de generar más ingresos. Estas dos condiciones hacen que el acceso a cuido tenga un efecto mucho mayor, en términos de horas trabajadas, que bajo condiciones menos atenuantes como las que enfrentan el resto de quintiles por su mejor situación socioeconómica. 


\section{CONCLUSIONES}

En esta investigación se estimó el efecto de dos programas de cuido (Hogares de Cuido y CECUDI) sobre la participación laboral y las horas trabajadas en las mujeres de los hogares beneficiados en Costa Rica. Para ello, se construyeron grupos de control similares utilizando diferentes métodos. Luego, con la muestra resultante, se hicieron estimaciones de los impactos utilizando métodos de MCO, logit binomial, y tobit. También se hicieron diferentes pruebas de robustez.

Se encontró un efecto significativo sobre la probabilidad de que la mujer participe en el mercado laboral, el efecto estimado está entre los 9 y 14 puntos porcentuales adicionales. En segundo lugar, se encontró que hay un impacto significativo, sobre las horas trabajadas entre las mujeres ocupadas. Se estima que los aumentos de horas trabajadas estarían entre el 8 y el 19 por ciento. Los resultados fueron robustos cuando se utilizaron modelos linéales y no lineales; así como ante cambios en las variables control.

Además, se encontró que los efectos en participación son más fuertes para las mujeres que no han completado la secundaria. También, hay evidencia parcial que sugiere que los efectos sobre las horas trabajadas son especialmente fuertes para el quintil más pobre de la distribución.

Estos resultados nos permiten concluir que la red de cuido, al aumentar la participación laboral de las mujeres, ha tenido un impacto positivo en los ingresos percibidos en los hogares de las mujeres beneficiadas, $y$ sobre todo de aquellos en un estado de mayor vulnerabilidad. Dado que los hogares beneficiados son de los quintiles con ingresos más bajos de la economía, es probable que esta política también haya tenido efectos en los niveles de pobreza de los hogares de estas mujeres y en general sobre el bienestar de sus hogares. Por lo tanto, para combatir la pobreza proveer de servicios de cuido es esencial; ya que los hogares pobres poseen en promedio más menores. Además, los hogares jefeados por una mujer que podrían beneficiarse más de estas políticas, tienen una mayor incidencia de pobreza (Robalino et al., 2019).

Así mismo, nuestros resultados implican que la red de cuido, si aumentara su cobertura, tiene el potencial de aumentar en forma significativa la oferta de trabajo de las mujeres en Costa Rica, tanto desde el margen extensivo (participación) como en el margen intensivo (aumento de horas entre las mujeres ocupadas). Esto tendría un impacto positivo sobre la economía, debido a que habría un aumento en las cantidades de mano de obra transadas $y$, por lo tanto, aumentos en la producción del país.

Cabe también mencionar las limitaciones del estudio. A pesar del trabajo minucioso que se realizó para poder controlar las diferencias entre las mujeres que reciben y no reciben cuido, todavía sigue siendo probable la presencia de elementos no observables que tengan el potencial de sesgar los resultados. Solamente un diseño experimental tiene el potencial de resolver la presencia de elementos no observables. Futuras investigaciones podrían utilizar diseños experimentales para poder verificar los resultados de este estudio.

Otra limitación del estudio es que el grupo de mujeres que actualmente son beneficiarias del programa podrían tener características diferentes al resto de mujeres en pobreza. Esto haría que la validez externa de nuestro estudio podría verse limitada. Dadas las pocas observaciones de beneficiarias del programa en la $\mathrm{ENAHO}^{8}$, no se espera que sea un resultado representativo $y$, por lo tanto, no se utilizan los factores de expansión. Cuando exista una expansión del programa de tal forma que aumente el número de observaciones en la encuesta y que los resultados tengan el potencial de ser representativos a nivel nacional, sería importante volver a hacer estas estimaciones.

8 Es a partir del 2016 que la encuestas ENAHO se plantea la misma pregunta asociada a los CECUDI y HC 


\section{AGRADECIMIENTOS}

Este artículo nace a partir de la tesis de licenciatura de Anibal Quiros. Se agradece profundamente los comentarios detallados a este artículo y a versiones previas de Laura Blanco y Andrea Collado. Además se agradece los comentarios de Isabel Cristina Araya, Ana María Carmiol, Marcela Román y de los pares anónimos. Juan Robalino agradece también el financiamiento de UCREA a través del proyecto "Intervenciones en Infancia Temprana para reducir la desigualdad en las oportunidades educativas" coordinado por la Dra. Ana María Carmiol.

\section{REFERENCIAS}

Alzúa, M. L., Cruces, G., \& Ripani, L. (2013). Welfare programs and labor supply in developing countries: experimental evidence from Latin America. Journal of Population Economics, 26(4), 1255-1284. https://doi.org/10.1007/s00148-012-0458-0

Ángeles, G., Gadsend, P., Galiani, S., Gertler, P., Herrera, A., Kariger, P., \& Seira, E. (2011). Evaluación de impacto del programa Estancias infantiles para apoyar a Madres Trabajadoras. (Informe final de la evaluación de impacto). CIEE e INSP, México.

Angrist, J. D., \& Pischke, J. S. (2008). Mostly harmless econometrics: An empiricist's companion. Princeton University Press.

Asamblea Legislativa de la República de Costa Rica. (1996, 23 de enero). Ley $N^{\circ} 7654$ de Pensiones Alimentarias. La Gaceta.

Ashenfelter, O. \& Heckman, J. (1974), The estimation of income and substitution effects in a model of family labour supply. Econométrica, 42(1), 73-85. https://doi.org/10.2307/1913686

Attanasio, O., \& Vera-Hernández, M. (2004). Medium-and long run effects of nutrition and child care: evaluation of a community nursery programme in rural Colombia. (IFS Working Papers, EWP04/06). The Institute for Fiscal Studies, Londres

Baker, M., Gruber, J., \& Milligan, K. (2008). Universal child care, maternal labor supply, and family well-being. Journal of political Economy, 116(4), 709-745. https://doi.org/10.1086/591908

Banco Mundial (2020). Labor force participation rate, female. Base de datos. Banco Mundial, Washington, DC. https://data.worldbank.org/indicator/SL.TLF.ACTI.FE.ZS

Becker, G. S. (1965). A Theory of the Allocation of Time. The Economic Journal, 75(299), 493-517. https://doi.org/10.2307/2228949

Beneria, L. (2007). The crisis of care, globalization of reproduction, and 'reconciliation' policies. (8th International GEM-IWG Conference: Engendering Macroeconomics and International Economics). Salt Lake: GEM-IWG and The Levy Economics Institute.

Berlinski, S., \& Galiani, S. (2007). The effect of a large expansion of pre-primary school facilities on preschool attendance and maternal employment. Labour Economics, 14(3), 665-680. https:// doi.org/10.1016/j.labeco.2007.01.003

Behrman, J. R., Cheng, Y., \& Todd, P. E. (2004). Evaluating preschool programs when length of exposure to the program varies: A nonparametric approach. Review of economics and statistics, 86(1), 108-132.

Borjas, G. (1996). Labor economics. MacGraw-Hill.

Cañas Calvo, M. L. C., Mora Mesén, G. M., Navarro Monge, A. E., Ruiz Quesada, E. M., Solano Aguilar, R. de los Á., \& Usaga Vargas, D. M. (2011). Programas no formales para la atención integral a la niñez en costa rica: Aciertos y limitaciones. Actualidades Investigativas en Educación, 7(3). https://doi.org/10.15517/aie.v7i3.9283 
Chinchilla Cerdas, S. (2017, 31 de julio). IMAS congeló espacios de niños en Red de Cuido por falta de presupuesto. La Nación. https://www.nacion.com/el-pais/politica/imas-congelo-espaciosde-ninos-en-red-de-cuido-por-falta-de-presupuesto/W6YKWRBLSRCLHGTP4NRCPC7VQQ/ story/

Chinchilla Cerdas, S. (2019, 5 de enero). Familias de clase media pueden acceder a Red de Cuido pagando el servicio. La Nación. https://www.nacion.com/el-pais/politica/familias-de-clasemedia-pueden-acceder-a-red-de/GH2FE62PMNHGPDEPJRR76UEX5Y/story/

Contraloría General de la República. (2011). Informe sobre el diseño y la implantación de la Red Nacional de Cuido y Desarrollo Infantil. (Informe No DFOE-SOC-IF-05-2011División de fiscalización operativa y evaluativa, área de servicios sociales. https://cgrfiles.cgr.go.cr/ publico/docs_cgr/2011/SIGYD_D_2011008060.pdf

Contraloría General de la República. (2015). Auditoría de carácter especial sobre la red nacional de cuido y desarrollo infantil. (Informe $\mathrm{N}^{0}$ DFOE-SOC-IF-18-2015).División de Fiscalización Operativa y Evaluativa, Área de Fiscalización de Servicios, Contraloría General de la República.

Deaton, A. (1985). Panel data from time series of cross sections. Journal of Econometrics, 30(1-2), 109-126. https://doi.org/10.1016/0304-4076(85)90134-4

Dehejia, R. H., \& Wahba, S. (2002). Propensity Score-Matching Methods for Nonexperimental Causal Studies. Review of Economics and Statistics, 84(1), 151-161. https://doi. org/10.1162/003465302317331982

Deutsch, R. (1998). Does Child Care Pay? Labor Force Participation and Earnings: Effects on Access to Child Care in the Favelas of Rio de Janeiro. (Working Paper No. 384). Banco Interamericano de Desarrollo.

Domingo, T. (2011). ¿Qué motiva a las mujeres a participar en el mercado laboral en periodos de crisis económica?. (IX Jornadas de Economía Laboral). Santiago de Compostela.

Encina Galaz, J., \& Martínez Alvear, C. (2009). Efecto de una mayor cobertura de salas cuna en la participación laboral femenina: evidencia de Chile. (Serie Documentos de Trabajo, No. 303). Universidad de Chile. http://repositorio.uchile.cl/handle/2250/144070

Ferrada Bórquez, L. M., \& Zarzosa Espina, P. (2010). Participación laboral de las mujeres en las regiones de Chile . Revista Universum, 25(2), 79-99. https://doi.org/10.4067/S071823762010000200006

Fondo de Desarrollo Social y Asignaciones Familiares. (2017). Ficha descriptiva CEN-CINAI, PANI y IMAS. Programas Sociales Selectivos. http://fodesaf.go.cr/buscador/Default. aspx?tipoBuscador $=2$

Fondo de Desarrollo Social y Asignaciones Familiares. (2018). Lineamientos generales de los Programas Sociales Financiados por el FODESAF. https://www.fodesaf.go.cr/acerca_del_ fodesaf/desaf/Plan_Presupuesto_de_los_programas_sociales.html

Gay, V., Hicks, D. L., Santacreu-Vasut, E. \& Shoham, A. (2018). Decomposing culture: An analysis of gender, language, and labor supply in the household. Review of Economics of the Household, 16(4), 879-909. https://doi.org/10.1007/s11150-017-9369-x

Goldin, C., Katz, L. F., \& Kuziemko, I. (2006). The homecoming of American college women: The reversal of the college gender gap. Journal of Economic Perspectives, 20(4), 133-156. https:// doi.org/10.1257/jep.20.4.133

Grillo, M., León, A. T., Mora, A., \& Rodríguez, I. (2010). Perspectivas Estratégicas y Técnicas sobre La Red nacional de Cuido y desarrollo Infantil. Propuesta de las Organizaciones sociales que Trabajan en el Sector de Niñez. (Documento de Trabajo). UNICEF Costa Rica. 
Haan, P., \& Wrohlich, K. (2009). Can child care policy encourage employment and fertility? Evidence from a structural model. (IZA Discussion Papers, No. 4503). Institute of Labor Economics

Instituto Mixto de Ayuda Social (2017). Estados Financieros y Opinión de los Auditores Independientes (2010-2017). (Informe final). Instituto Mixto De Ayuda Social Y Dirección Empresas Comerciales.

Instituto Mixto de Ayuda Social . (2018). Informe del Programa Protección y Promoción Social (2017). Subgerencia de Desarrollo Social Sistemas de Información. https://www.imas. go.cr/sites/default/files/docs/Informe\%20Programa\%20de\%20Promoci\%C3\%B3n\%20y\%20 Protecci\%C3\%B3n\%20Social\%20del\%202019.pdf

Instituto Mixto de Ayuda Social. (2020). Objetivos CECUDI. https:/web.archive.org/ web/20170725182906/http://www.imas.go.cr:80/beneficios/cuido-y-desarrollo-infantil-0

Instituto Nacional de Estadística y Censos. (2010;2019). Encuesta Nacional de Hogares (ENAHO). https://www.inec.cr/encuestas/encuesta-nacional-de-hogares

Jiménez-Fontana, P. (2016). Retos para materializar el dividendo de género perfiles de uso de tiempo en Costa Rica. Población y Salud en Mesoamérica , 13(2), 1-23. https://doi.org/10.15517/psm. v13i2.21748

Kam, C. D., \& Palmer, C. L. (2008). Reconsidering the Effects of Education on Political Participation. The Journal of Politics, 70(3), 612-631. https://doi.org/10.1017/ s0022381608080651

Goraus, K., \& Tyrowicz, J. (2014). Gender Wage Gap in Poland-Can it be explained by Differences in Observable Characteristics?. (Working Papers Series of the Faculty of Economic Sciences, No. 11/2014 (128)). University of Warsaw.

Lang Clachar, G., Soto Méndez, B., \& Robalino, J. (2015). Efecto del programa “Avancemos" sobre el trabajo infantil en Costa Rica. (Serie de documentos de trabajo, No. 15-03). Escuela de Economía, Universidad de Costa Rica.

Lovász, A., \& Szabó-Morvai, A. (2013). Does childcare matter for maternal labor supply? Pushing the limits of the regression discontinuity framework. (Budapest Working Papers on the Labour Market, No. BWP - 2013/13). Institute of Economics, Hungarian Academy of Sciences.

Mateo Diaz, M., \& Rodriguez-Chamussy, L. (2016). Cashing in on education: Women, childcare, and prosperity in Latin America and the Caribbean. World Bank; Inter-American Development Bank. https://doi.org/10.1596/978-1-4648-0902-6

Mayer, A. K. (2011). Does Education Increase Political Participation?. The Journal of Politics, 73(3), 633-645. https://doi.org/10.1017/s002238161100034x

McConnel, C. R., Brue, S. L., \& Macpherson, D. A. (2003), Economía Laboral. (E. Rabasco, Trad.; Sexta edición). McGraw-Hill.

Ministerio de Trabajo y Seguridad Social. (2016). Informe de Estudio Especial Programa Red Nacional de Cuido y Desarrollo Infantil, Financiado con Recursos del Fondo de Desarrollo Social y Asignaciones Familiares -FODESAF-. (INFORME AU-IF-FOD-EE-00017-2016). Ministerio de Trabajo y Seguridad Social, Costa Rica.

Nollenberger, N., \& Perazzo, I. (2016). Efectos de la provisión universal de educación preescolar sobre la asistencia y la participación laboral femenina. Evidencia para el caso uruguayo. (Serie Documentos de Trabajo, DT 04/2016), Universidad de la República, Montevideo. https:// hdl.handle.net/20.500.12008/19012

Organización de Estado Iberoamericanos. (2018). Educación Inicial Experiencias no Escolarizadas. Costa Rica. https://web.archive.org/web/20180515203738/http://www.oei.es/historico/inicial/ costaricane.htm 
Porras, M. E. (2012). Aumento de la participación de la mujer en el mercado laboral y sus efectos en actividades asignadas por tradición: el cuido a niños y niñas y de los adultos mayores. XVII Congreso Internacional del CLAD sobre la Reforma del Estado y de la Administración Pública, Cartagena, Colombia.

Programa Estado de la Nación. (2010). Decimosexto Informe Estado de la Nación en Desarrollo Humano Sostenible. http://hdl.handle.net/20.500.12337/904

Programa del Estado de la Nación. (2015). Capítulo 2: Equidad e integración social. En Vigésimo primer Informe Estado de la Nación en Desarrollo Humano Sostenible. http://hdl.handle. net/20.500.12337/82

Programa Estado de la Nación (2018). Informe Estado de la Nación 2018. http://hdl.handle. net/20.500.12337/2983

Programa Estado de la Nación (2019). Informe Estado de la Nación 2019. http://hdl.handle. net/20.500.12337/7808

Ramos-Chaves, A. R. (2010). Essays on Economic Development in Costa Rica [Doctoral dissertation, UC Berkeley]. https://escholarship.org/uc/item/28j1w8df

Robalino, J. A., Paredes, S., Mayorga, B., Cordoba, D., \& Blanco, L., (2019). Informe sobre la evolución del mercado laboral y la pobreza en Costa Rica, Tendencias 2010-2016. Escuela de Economía de la Universidad de Costa Rica.

Rosero, J., \& Oosterbeek, H. (2011). Trade-offs between Different Early Childhood Interventions: Evidence from Ecuador. (Discussion Papers, No. TI 2011-102/3). Tinbergen Institute .

UNICEF Costa Rica (2020, 28 de abril). Más de 60.000 niñas y niños se verán beneficiados con nuevo plan de fortalecimiento a la Red Nacional de Cuido y Desarrollo Infant. https://www.unicef. org/costarica/comunicados-prensa/60000-ni\%C3\%B1as-y-ni\%C3\%B1os-se-ver\%C3\%A1nbeneficiados-con-nuevo-plan-de

Universidad de Costa Rica-CICAP-ESP. (2016). Informe Final. Evaluación del diseño, gestión y ejecución del beneficio "Alternativas de Atención y Desarrollo de la Niñez", que atienden a los niños y a las niñas que reciben el subsidio del IMAS en el marco del Programa Red Nacional de Cuido y Desarrollo. UCR-CICAP-ESP. 